\title{
Recent trends in chronic disease, impairment and disability among older adults in the United States
}

William W Hung ${ }^{1,2^{*}}$, Joseph S Ross ${ }^{3}$, Kenneth S Boockvar ${ }^{1,2}$ and Albert L Siu ${ }^{1,2}$

\begin{abstract}
Background: To examine concurrent prevalence trends of chronic disease, impairment and disability among older adults.

Methods: We analyzed the 1998, 2004 and 2008 waves of the Health and Retirement Study, a nationally representative survey of older adults in the United States, and included 31,568 community dwelling adults aged 65 and over. Measurements include: prevalence of chronic diseases including hypertension, heart disease, stroke, diabetes, cancer, chronic lung disease and arthritis; prevalence of impairments, including impairments of cognition, vision, hearing, mobility, and urinary incontinence; prevalence of disability, including activities of daily living (ADLs) and instrumental activities of daily living (IADLS).

Results: The proportion of older adults reporting no chronic disease decreased from 13.1\% (95\% Confidence Interval [Cl], 12.4\%-13.8\%) in 1998 to $7.8 \%(95 \% \mathrm{Cl}, 7.2 \%-8.4 \%)$ in 2008, whereas the proportion reporting 1 or more chronic diseases increased from 86.9\% (95\% Cl, 86.2\%-89.6\%) in 1998 to $92.2 \%$ (95\% Cl, 91.6\%-92.8\%) in 2008. In addition, the proportion reporting 4 or more diseases increased from $11.7 \%(95 \% \mathrm{Cl}, 11.0 \%-12.4 \%)$ in 1998 to $17.4 \%$ (95\% Cl, 16.6\%-18.2\%) in 2008. The proportion of older adults reporting no impairments was $47.3 \%$ (95\% Cl, 46.3\%48.4\%) in 1998 and $44.4 \%(95 \% \mathrm{Cl}, 43.3 \%-45.5 \%)$ in 2008, whereas the proportion of respondents reporting 3 or more was $7.2 \%(95 \% \mathrm{Cl}, 6.7 \%-7.7 \%)$ in 1998 and $7.3 \%(95 \% \mathrm{Cl}, 6.8 \%-7.9 \%)$ in 2008. The proportion of older adults reporting any $\mathrm{ADL}$ or IADL disability was $26.3 \%(95 \% \mathrm{Cl}, 25.4 \%-27.2 \%)$ in 1998 and $25.4 \%$ (95\% Cl, 24.5\%-26.3\%) in 2008.
\end{abstract}

Conclusions: Multiple chronic disease is increasingly prevalent among older U.S. adults, whereas the prevalence of impairment and disability, while substantial, remain stable.

Keywords: chronic disease, impairment, disability, prevalence trends

\section{Background}

Disability, such as the inability to dress, bathe or manage one's medications, is prevalent and costly among older adults in the United States. The development of such disability among older adults is often complex and multifactorial [1-3]. Many have postulated that co-morbid chronic diseases are significant risk factors for developing disabilities [1]. Over the past 20 years, there has been a rise in chronic disease prevalence $[4,5]$, and the majority of the older population over age 65 now suffers from multiple chronic diseases $[4,6,7]$. However, recent

\footnotetext{
* Correspondence: william.hung@mssm.edu

'Department of Geriatrics and Palliative Medicine, Mount Sinai School of Medicine, New York, NY, USA

Full list of author information is available at the end of the article
}

literature on the patterns of chronic diseases and disability found that despite the increase in the prevalence of chronic diseases, disability prevalence has declined [8-14], although not uniformly across groups of different age and sex [15-17]. Populations in other developed countries, such as the Netherlands, have also observed increases in chronic disease prevalence but stable or declines in disability rates $[18,19]$. However, trends in disability rates among developed countries are not uniformly stable or in decline, as Belgium, Japan and Sweden reported increases in disability [20].

Considering the pathway to disability, impairments have been considered an intermediary between chronic diseases and disability [1,2]. For example, a person with chronic arthritis may develop mobility impairment; or a

\section{() Biomed Central}


person with diabetes mellitus may develop diabetic retinopathy which impairs vision. In turn, the mobility or visually impaired person may become unable to carry out tasks which are essential for independent living. Prior examinations of individual impairments-such as impairments in vision, hearing and cognition [21-23] have suggested that they may have become less prevalent over time, but other studies have contradicted these findings, demonstrating that there were no significant changes $[24,25]$. The patterns of impairments, which disproportionately affect older adults, and multiple impairments over time are not well known. Given the dynamics between chronic disease, impairment and disability, and that significant changes have occurred recently in terms of socio-demographic makeup and health care among older adults, an up to date examination of the trends of impairments and disability among older adults may improve our current understanding of health care needs for this population.

Therefore our primary objective is to examine the trends of impairments among older adults in the context of increasing trends of chronic diseases, and opposing trends of disability, in order to better describe the health care needs of older adults. A second objective is to use the most recent data to examine the continuing detailed trends of chronic diseases and disability stratified by age and sex. The Health and Retirement Study (HRS) [26], which is a nationally representative survey of older adults in the US, surveys adults on multiple impairments and disability, and thus, provides an excellent opportunity to examine these concurrent trends among older adults.

\section{Methods}

The Health and Retirement Study (HRS) [26] is a national longitudinal survey of U.S. adults over age 50 sponsored by the National Institute on Aging and conducted by the Institute for Social Research at the University of Michigan. The study was designed to investigate the experience of aging among older adults as they advance from work to retirement with particular emphasis on health insurance, savings, and trajectories of economic and physical well-being. The initial wave of the HRS conducted in 1992 comprised the core sample of the HRS and included 12,652 community-dwelling adults between the ages of 51 and 61 or their spouses, regardless of age, collected via in-home interviews. The sample was combined in 1998 with the Asset and Health Dynamics among the Oldest-Old Study (AHEAD) $[27,28]$, which is a survey of a nationally representative sample of persons who were born in 1923 or before. Subsequent waves were supplemented with new birth cohorts every 6 years so as to be representative of the entire US household population aged 50 and above. A full description of the procedures used in the HRS surveys has been published previously [26]. The survey data are publicly available and do not contain any unique identifiers. Data from three waves of interviews were collated for the present analysis: 1998, 2004 and 2008. We only included older adults who were at least 65 years of age at the times of the interview.

\section{Chronic Disease Definition}

In each wave, respondents were asked to self-report whether they had ever been told by their physician that they had any of several chronic diseases. Proxy respondents were surveyed if the respondents were not able to participate in the survey. Chronic diseases surveyed in all three waves of the HRS studied included hypertension, heart conditions (which included coronary heart diseases and heart failure), chronic lung disease (such as chronic bronchitis or emphysema, but not asthma), diabetes, stroke (which also included transient ischemic attack), cancer (or a malignant tumor of any kind except skin cancer), and arthritis. We then categorized respondents by whether they self-reported $0,1,2,3$ and 4 or more chronic diseases.

\section{Impairment Definition}

In each wave, respondents were asked to self-report whether they had any of several impairments. We used a similar approach as Cigolle et al [29] in defining impairments and included several impairments selfreported by respondents: (1) vision (fair or poor eyesight or blindness despite the use of glasses or corrective lenses as usual); (2) hearing (fair or poor hearing despite the use of a hearing aid as usual); and (3) cognition. Cognition was measured in each wave using a standardized test on a 35 point scale [30], with higher scores indicating better cognitive function. This scale was modified from the Telephone Interview for Cognitive Status and contained similar items to the Mini Mental Status Examination [31], and has been validated as a cognitive screening instrument. We defined cognitive impairment as a score at or below 8, which has been previously used as cutoff score for severe cognitive impairment because it was consistent with other estimates of the prevalence of dementia [32]. In addition, for respondents who were unable to complete the survey and had proxy respondents, proxy reports of fair or poor memory were considered to be indicative of cognitive impairment of the respondent, as has been done in prior research [33].

We further included two other impairments commonly seen in older adults: urinary incontinence and mobility impairment. Respondents with loss of control of their urination in the past 12 months were characterized to have urinary incontinence. Prior national survey data such as the National Health and Nutrition 
Examination Survey (NHANES) also use similar definition for estimating the prevalence of urinary incontinence [34]. Respondents who reported difficulty in ambulating across the room were characterized as having mobility impairment. This measure has previously been used in other large scale studies such as the Women's Health and Aging Study as a marker for severe mobility difficulty [35]. In total, we characterized 5 conditions as indicative of impairment (visual, hearing, cognitive, mobility impairment and urinary incontinence). We then categorized respondents by whether they self-reported $0,1,2$, and 3 or more impairments.

\section{Disability Definition}

In each wave, respondents were asked to self-report whether they had any difficulty with any of several activities of daily living (ADL) and instrumental activities of daily living (IADL). The following ADLs were surveyed in each of the three survey waves: bathing, dressing, eating, toileting and transferring; and the following IADLs were surveyed: using the telephone, managing money, managing medications, grocery shopping and preparation of meals. We characterized respondents as having disability in a task if they reported difficulty, or received help for the task, or could not perform the task secondary to health reasons. We then categorized respondents by whether they self-reported any disability in ADL tasks, in IADL tasks, and in either ADL or IADL tasks.

\section{Other Variables}

Several other socio-demographic variables were included in our analyses for the purposes of describing changes in population characteristics: age, sex, race, marital status and education. We categorized race into categories of white and non-white, marital status into categories of married and not married, and education into categories of under $8^{\text {th }}$ grade education, $8^{\text {th }}$ to $11^{\text {th }}$ grade education, high school education, and beyond high school education. We also included body mass index and current smoking status as indicators of body size and health behaviors.

\section{Statistical Analysis}

Respondent characteristics were summarized for each wave of the HRS: 1998, 2004, and 2008. Next we described the prevalence of each chronic disease, impairment and disability in each wave, and summarized the prevalence of multiple chronic diseases and multiple impairments in each wave. We examined the concurrent trends of multiple chronic diseases, impairments and disability across all three survey years. We then stratified each sample into age groups of 65-69, 70-74, 75-79, 8084 and 85 and above and by sex, and examined the proportions of respondents with different numbers of chronic diseases, impairments, and disability. The purpose of stratifying in 5 year groups is to (1) examine whether there are age dependent trends and (2) to clarify that the changes in chronic disease, impairment, and disability prevalence were not due to the accumulation of diseases in the same cohort over time. We performed sensitivity analysis by removing each individual chronic disease and impairment from the total number of conditions to test if observed trends were due to one condition only. We used weighted chi square test with Wald distribution to test group differences in proportions and one way ANOVA F-test for continuous variables. We used a p-value threshold of 0.05 for statistical significance. We applied sampling weights according to the methodology described in the HRS manual for the application of sampling weights for respondent level characteristics [36]. The respondent level weight is nonzero for living non-institutionalized respondents in the appropriate years. It is scaled so as to yield weight sums which correspond to the number of individuals in the U.S. population as measured by the March Current Population Survey (CPS) for the year of data collection. All analyses were performed using Stata version 8.0 (Stata Corp LP, College Station, TX). Because the HRS is a publicly available anonymous data source, our study was exempted from review by the Mount Sinai Institutional Review Board.

\section{Results}

A total of 10,390,10,621 and 10,557 respondents were included in the HRS waves in 1998, 2004 and 2008, respectively. Among all three survey waves, the average age was $74.6,57.3 \%$ were female, $89.1 \%$ were white, and $55.1 \%$ were married (Table 1 ) and there were no clinically significant differences in the age, sex, race, and marital status across all three survey years. However, education levels and average BMI rose in subsequent survey years, from $33.1 \%$ to $40.3 \%$ completing an education above high school and from 26.0 to $27.5 \mathrm{~kg} / \mathrm{m}^{2}$ respectively.

\section{Chronic Disease Trends}

Among the chronic diseases examined, nearly all diseases, including hypertension, diabetes, cancer, chronic lung disease and arthritis showed an increasing trend throughout the study period (Table 1). On the other hand, the prevalence of heart conditions increased initially from 1998 to 2004 and subsequently remained stable as of 2008. The proportion of respondents reporting varying number of chronic diseases changed over the study period (Table 2 and Figure $1 \mathrm{~A}$ in the online supplement; $\mathrm{p}$ value $<0.001)$. The proportion reporting no chronic disease decreased from $13.1 \%$ in 1998 to $8.8 \%$ in 2004 to $7.8 \%$ in 2008 , as did the proportion reporting 1 chronic disease, from 26.9\% in 1998 to 
Table 1 Descriptive statistics of adults aged 65 and over in the Health and Retirement Study (HRS) in 1998, 2004 and 2008

\begin{tabular}{|c|c|c|c|c|c|}
\hline Demographic characteristics & Overall & $\begin{array}{c}1998 \\
(n=10390)\end{array}$ & $\begin{array}{c}2004 \\
(n=10621)\end{array}$ & $\begin{array}{c}2008 \\
(n=10557)\end{array}$ & p-value \\
\hline Mean age, y $(95 \% \mathrm{Cl})$ & $\begin{array}{c}74.6 \\
(74.5,74.7)\end{array}$ & $\begin{array}{c}74.4 \\
(74.3,74.5)\end{array}$ & $\begin{array}{c}74.9 \\
(74.7,75.0)\end{array}$ & $\begin{array}{c}74.5 \\
(74.3,74.6)\end{array}$ & $<0.001$ \\
\hline Female sex, \% (95\%Cl) & $\begin{array}{c}57.3 \\
(56.7,57.9)\end{array}$ & $\begin{array}{c}58.1 \\
(57.0,59.1)\end{array}$ & $\begin{array}{c}57.1 \\
(56.0,58.1)\end{array}$ & $\begin{array}{c}56.9 \\
(55.9,58.0)\end{array}$ & 0.26 \\
\hline Race & & & & & 0.40 \\
\hline White, \% (95\% Cl) & $\begin{array}{c}89.5 \\
(89.2,89.8)\end{array}$ & $\begin{array}{c}89.4 \\
(88.8,89.9)\end{array}$ & $\begin{array}{c}89.8 \\
(89.3,90.4)\end{array}$ & $\begin{array}{c}89.4 \\
(88.8,90.0)\end{array}$ & \\
\hline Black, \% (95\% Cl) & $\begin{array}{c}8.3 \\
(8.0,8.6)\end{array}$ & $\begin{array}{c}8.5 \\
(8.0,9.0)\end{array}$ & $\begin{array}{c}8.1 \\
(7.6,8.6)\end{array}$ & $\begin{array}{c}8.3 \\
(7.9,8.8)\end{array}$ & \\
\hline Other, \% (95\% Cl) & $\begin{array}{c}2.2 \\
(2.0,2.4)\end{array}$ & $\begin{array}{c}2.1 \\
(1.8,2.4)\end{array}$ & $\begin{array}{c}2.1 \\
(1.8,2.4)\end{array}$ & $\begin{array}{c}2.3 \\
(2.0,2.6)\end{array}$ & \\
\hline Married, \% (95\% Cl) & $\begin{array}{c}55.1 \\
(54.4,55.7) \\
\end{array}$ & $\begin{array}{c}55.3 \\
(54.3,56.4)\end{array}$ & $\begin{array}{c}55.9 \\
(54.9,56.9)\end{array}$ & $\begin{array}{c}54.1 \\
(53.0,55.2)\end{array}$ & 0.05 \\
\hline Education, \% (95\% Cl) & & & & & $<0.001$ \\
\hline Under $8^{\text {th }}$ grade & $\begin{array}{c}7.5 \\
(7.2,7.8)\end{array}$ & $\begin{array}{c}9.6 \\
(9.1,10.2)\end{array}$ & $\begin{array}{c}7.1 \\
(6.6,7.6)\end{array}$ & $\begin{array}{c}6.0 \\
(5.6,7.5)\end{array}$ & \\
\hline 8-11 grade & $\begin{array}{c}20.6 \\
(20.1,21.1)\end{array}$ & $\begin{array}{c}24.2 \\
(23.4,25.1)\end{array}$ & $\begin{array}{c}20.2 \\
(19.4,21.1)\end{array}$ & $\begin{array}{c}18.0 \\
(20.1,21.1)\end{array}$ & \\
\hline High school & $\begin{array}{c}34.9 \\
(34.4,35.5)\end{array}$ & $\begin{array}{c}33.1 \\
(32.1,34.1)\end{array}$ & $\begin{array}{c}35.8 \\
(34.8,36.8)\end{array}$ & $\begin{array}{c}35.7 \\
(34.4,35.5)\end{array}$ & \\
\hline Above high school & $\begin{array}{c}37.0 \\
(36.4,37.6)\end{array}$ & $\begin{array}{c}33.1 \\
(32.1,34.1)\end{array}$ & $\begin{array}{c}36.9 \\
(35.9,37.9)\end{array}$ & $\begin{array}{c}40.3 \\
(36.4,37.6)\end{array}$ & \\
\hline Current Smoker, \% (95\% Cl) & $\begin{array}{c}10.1 \\
(9.7,10.4)\end{array}$ & $\begin{array}{c}10.9 \\
(10.3,11.6)\end{array}$ & $\begin{array}{c}9.3 \\
(8.8,10.0)\end{array}$ & $\begin{array}{c}10.1 \\
(9.4,10.8)\end{array}$ & 0.002 \\
\hline Body Mass Index, (95\% Cl) & $\begin{array}{c}26.7 \\
(26.6,26.8)\end{array}$ & $\begin{array}{c}26.0 \\
(25.9,26.1)\end{array}$ & $\begin{array}{c}26.6 \\
(26.4,26.7)\end{array}$ & $\begin{array}{c}27.5 \\
(27.3,27.6)\end{array}$ & $<0.001$ \\
\hline \multicolumn{6}{|l|}{ Chronic diseases, \% } \\
\hline \multicolumn{6}{|l|}{$(95 \% \mathrm{Cl})$} \\
\hline Hypertension & $\begin{array}{c}59.7 \\
(59.2,60.3)\end{array}$ & $\begin{array}{c}52.5 \\
(51.5,53.5)\end{array}$ & $\begin{array}{c}60.5 \\
(59.5,61.5)\end{array}$ & $\begin{array}{c}65.0 \\
(63.9,66.0)\end{array}$ & $<0.001$ \\
\hline Heart conditions & $\begin{array}{c}31.4 \\
(30.8,32.0)\end{array}$ & $\begin{array}{c}30.7 \\
(29.7,31.6)\end{array}$ & $\begin{array}{c}31.9 \\
(30.9,32.9)\end{array}$ & $\begin{array}{c}31.6 \\
(30.6,32.5)\end{array}$ & 0.20 \\
\hline Stroke & $\begin{array}{c}9.3 \\
(9.0,9.7)\end{array}$ & $\begin{array}{c}9.8 \\
(9.2,10.4)\end{array}$ & $\begin{array}{c}8.9 \\
(8.4,9.6)\end{array}$ & $\begin{array}{c}9.2 \\
(8.6,9.8)\end{array}$ & 0.14 \\
\hline Diabetes & $\begin{array}{c}19.3 \\
(18.8,19.8)\end{array}$ & $\begin{array}{c}15.2 \\
(14.5,15.9)\end{array}$ & $\begin{array}{c}19.3 \\
(18.4,20.1)\end{array}$ & $\begin{array}{c}22.7 \\
(21.8,23.6)\end{array}$ & $<0.001$ \\
\hline Cancer & $\begin{array}{c}17.4 \\
(16.9,17.9)\end{array}$ & $\begin{array}{c}14.6 \\
(13.9,15.3)\end{array}$ & $\begin{array}{c}18.1 \\
(17.3,18.9)\end{array}$ & $\begin{array}{c}19.1 \\
(18.2,19.9)\end{array}$ & $<0.001$ \\
\hline Chronic lung disease & $\begin{array}{c}11.6 \\
(11.2,12.0)\end{array}$ & $\begin{array}{c}10.8 \\
(10.1,11.4)\end{array}$ & $\begin{array}{c}11.6 \\
(10.9,12.3)\end{array}$ & $\begin{array}{c}12.3 \\
(11.6,13.0)\end{array}$ & 0.01 \\
\hline Arthritis & $\begin{array}{c}65.5 \\
(64.9,66.1)\end{array}$ & $\begin{array}{c}59.1 \\
(58.1,60.1)\end{array}$ & $\begin{array}{c}67.7 \\
(66.7,68.6)\end{array}$ & $\begin{array}{c}68.8 \\
(67.8,69.8)\end{array}$ & $<0.001$ \\
\hline \multicolumn{6}{|l|}{ Impairments, \% } \\
\hline \multicolumn{6}{|l|}{$(95 \% \mathrm{Cl})$} \\
\hline $\begin{array}{l}\text { Severe Cognitive } \\
\text { Impairment }\end{array}$ & $\begin{array}{c}4.3 \\
(4.1,4.6)\end{array}$ & $\begin{array}{c}4.9 \\
(4.5,5.3)\end{array}$ & $\begin{array}{c}4.2 \\
(3.8,4.5)\end{array}$ & $\begin{array}{c}4.0 \\
(3.6,4.4)\end{array}$ & 0.01 \\
\hline Visual Impairment & $\begin{array}{c}23.7 \\
(23.2,24.2)\end{array}$ & $\begin{array}{c}25.4 \\
(24.5,26.3)\end{array}$ & $\begin{array}{c}23.6 \\
(22.7,24.5)\end{array}$ & $\begin{array}{c}22.4 \\
(21.6,23.3)\end{array}$ & $<0.001$ \\
\hline Hearing Impairment & $\begin{array}{c}25.8 \\
(25.3,26.4)\end{array}$ & $\begin{array}{c}25.4 \\
(24.5,26.3)\end{array}$ & $\begin{array}{c}27.2 \\
(26.2,28.1)\end{array}$ & $\begin{array}{c}25.0 \\
(24.1,25.9)\end{array}$ & 0.003 \\
\hline Urinary incontinence & $\begin{array}{c}23.8 \\
(23.3,24.4)\end{array}$ & $\begin{array}{c}19.8 \\
(19.0,20.6)\end{array}$ & $\begin{array}{c}23.6 \\
(22.7,24.4)\end{array}$ & $\begin{array}{c}27.5 \\
(26.5,28.4)\end{array}$ & $<0.001$ \\
\hline Mobility Impairment & $\begin{array}{c}8.2 \\
(7.9,8.6)\end{array}$ & $\begin{array}{c}8.3 \\
(7.8,8.9)\end{array}$ & $\begin{array}{c}7.9 \\
(7.3,8.4)\end{array}$ & $\begin{array}{c}8.5 \\
(7.9,9.1)\end{array}$ & 0.27 \\
\hline
\end{tabular}



2008 (Continued)

\begin{tabular}{|c|c|c|c|c|c|}
\hline \multicolumn{6}{|l|}{ Disability, \% (95\% } \\
\hline \multicolumn{6}{|l|}{$\mathrm{Cl})$} \\
\hline $\begin{array}{l}\text { ADL dependence } \\
\text { Bath }\end{array}$ & $\begin{array}{c}8.6 \\
(8.3,9.0)\end{array}$ & $\begin{array}{c}9.8 \\
(9.2,10.4)\end{array}$ & $\begin{array}{c}8.2 \\
(7.6,8.8)\end{array}$ & $\begin{array}{c}8.1 \\
(7.5,8.6)\end{array}$ & 0.001 \\
\hline Bed transfer & $\begin{array}{c}6.0 \\
(5.7,6.3)\end{array}$ & $\begin{array}{c}6.9 \\
(6.4,7.4)\end{array}$ & $\begin{array}{c}5.3 \\
(4.9,5.8)\end{array}$ & $\begin{array}{c}5.9 \\
(5.4,6.4)\end{array}$ & 0.001 \\
\hline $\begin{array}{l}\text { Dress } \\
\text { Eating }\end{array}$ & $\begin{array}{c}10.9 \\
(10.5,11.3) \\
3.8 \\
(3.6,4.1)\end{array}$ & $\begin{array}{c}11.5 \\
(10.8,12.1) \\
4.1 \\
(3.7,4.5)\end{array}$ & $\begin{array}{c}10.2 \\
(9.5,10.8) \\
3.7 \\
(3.3,4.1)\end{array}$ & $\begin{array}{c}11.0 \\
(10.4,11.7) \\
3.8 \\
(3.4,4.2)\end{array}$ & $\begin{array}{l}0.01 \\
0.28\end{array}$ \\
\hline Toileting & $\begin{array}{c}6.3 \\
(6.1,6.6)\end{array}$ & $\begin{array}{c}6.9 \\
(6.4,7.5)\end{array}$ & $\begin{array}{c}5.8 \\
(5.3,6.3)\end{array}$ & $\begin{array}{c}6.4 \\
(5.8,6.9)\end{array}$ & 0.01 \\
\hline \multicolumn{6}{|l|}{ IADL dependence } \\
\hline Meal preparation & $\begin{array}{c}7.6 \\
(7.2,7.9)\end{array}$ & $\begin{array}{c}7.8 \\
(7.2,8.3)\end{array}$ & $\begin{array}{c}7.5 \\
(7.0,8.1)\end{array}$ & $\begin{array}{c}7.4 \\
(6.9,8.0)\end{array}$ & 0.66 \\
\hline Medication management & $\begin{array}{c}4.0 \\
(3.8,4.2)\end{array}$ & $\begin{array}{c}3.9 \\
(3.5,4.2)\end{array}$ & $\begin{array}{c}3.9 \\
(3.5,4.3)\end{array}$ & $\begin{array}{c}4.2 \\
(3.8,4.6)\end{array}$ & 0.53 \\
\hline Money management & $\begin{array}{c}7.1 \\
(6.8,7.5)\end{array}$ & $\begin{array}{c}7.4 \\
(6.9,7.9)\end{array}$ & $\begin{array}{c}7.0 \\
(6.5,7.5)\end{array}$ & $\begin{array}{c}7.1 \\
(6.5,7.6)\end{array}$ & 0.56 \\
\hline Shopping & $\begin{array}{c}11.1 \\
(10.7,11.4)\end{array}$ & $\begin{array}{c}11.7 \\
(11.0,12.3)\end{array}$ & $\begin{array}{c}11.2 \\
(10.6,11.9)\end{array}$ & $\begin{array}{c}10.4 \\
(9.8,11.0)\end{array}$ & 0.02 \\
\hline Telephone use & $\begin{array}{c}5.6 \\
(5.3,5.9)\end{array}$ & $\begin{array}{c}5.9 \\
(5.5,6.4)\end{array}$ & $\begin{array}{c}5.6 \\
(5.1,6.1)\end{array}$ & $\begin{array}{c}5.6 \\
(4.8,5.7)\end{array}$ & 0.15 \\
\hline
\end{tabular}

Abbreviations: $\mathrm{Cl}$, confidence interval; $\mathrm{ADL}$, activities of daily living; IADL, instrumental activities of daily living.

Note: All estimates used sampling weights to account for survey design (with $95 \%$ confidence intervals in parentheses); $\mathrm{p}$ - values were derived from the Wald chi-square tests for categorical variables and ANOVA F-test for continuous variables for association between the proportion or characteristics of respondents and year of survey.

$23.1 \%$ in 2004 to $21.2 \%$ in 2008 . The proportion reporting 1 or more chronic diseases increased from $86.9 \%$ in 1998 to $91.2 \%$ in 2004 to $92.2 \%$ in 2008 . In contrast, the proportion reporting 3 chronic diseases increased from $19.1 \%$ in 1998 to $22.7 \%$ in 2004 to $23.9 \%$ in 2008 , as did the proportion reporting 4 or more chronic diseases, from $11.6 \%$ in 1998 to $15.2 \%$ in 2004 to $17.4 \%$ in 2008 .

\section{Impairment Trends}

Among impairments, the prevalence of hearing and mobility impairment remained stable, whereas the prevalence of visual and cognitive impairment declined slightly (Table 1). Urinary incontinence, however, increased in prevalence. The proportion of respondents reporting varying number of impairments changed over the study period (Table 3 and Figure $1 \mathrm{~B}$ in the online supplement; $\mathrm{p}=0.001$ ). The proportion of respondents reporting no impairments declined slightly from $47.3 \%$ in 1998 to $45.3 \%$ in 2004 and $44.4 \%$ in 2008 , whereas the proportion reporting 1 impairment increased slightly, from $31.0 \%$ in 1998 to $32.7 \%$ in 2004 to $33.1 \%$ in 2008. The proportion of respondents reporting 2 or 3 or more impairments remained stable.

\section{Disability Trends}

Among ADL and IADL disabilities, with the exception of money management, the prevalence of the various disabilities examined during the study time period remained stable (Table 1 ). The proportion of respondents reporting any ADL disability did not change consistently over the study period, from $19.2 \%$ in 1998 to $17.4 \%$ in 2004 , to $18.4 \%$ in 2008 (Table 4 and Figure $1 \mathrm{C}$ in the online supplement; $\mathrm{p}=0.01$ ), and there was a non-significant decline in the proportion reporting any IADL disability, from $17.8 \%$ in 1998 to $17.7 \%$ in 2004 to $16.6 \%$ in $2008(\mathrm{p}=0.06)$. The proportion of respondents reporting any ADL or IADL disability declined initially from $26.3 \%$ in 1998 to $25.3 \%$ in 2004 , but remained steady as of 2008 at $25.4 \%(p=0.23)$.

\section{Age-Stratified Trends}

For chronic disease trends, there was an increasing trend towards higher proportion of respondents with higher number of chronic diseases among all age groups (Table 2). For impairment trends, there were no consistent patterns observed among respondents in all age group except among those aged $65-69$, where the proportion with no impairment declined from $58.8 \%$ in 1998 to $53.0 \%$ in 2008 and the proportion of respondents with 1 and 2 impairments increased (Table 3). For disability trends, there was a significant decline in IADL disability $(\mathrm{p}<0.01)$ and any disability $(\mathrm{p}=0.02)$ among the oldest age group (85 and above), as well as in IADL disability among those aged 75-79 ( $\mathrm{p}<0.01$; Table 4). 
Table 2 Percentage of respondents with $0,1,2,3$, or 4 or more chronic diseases by survey year, overall and stratified by age and by sex

\begin{tabular}{|c|c|c|c|c|c|}
\hline & \multicolumn{5}{|c|}{ Number of chronic diseases, $\%$ (95\% Confidence Interval) } \\
\hline & 0 & 1 & 2 & 3 & $4+$ \\
\hline \multicolumn{6}{|l|}{ Overall* } \\
\hline $1998, n=10390$ & $\begin{array}{c}13.1 \\
(12.4,13.8)\end{array}$ & $\begin{array}{c}26.9 \\
(26.0,27.8)\end{array}$ & $\begin{array}{c}29.4 \\
(28.5,30.3)\end{array}$ & $\begin{array}{c}19.1 \\
(18.3,19.9)\end{array}$ & $\begin{array}{c}11.6 \\
(10.9,12.2\end{array}$ \\
\hline $2004, n=10621$ & $\begin{array}{c}8.8 \\
(8.2,9.4)\end{array}$ & $\begin{array}{c}23.1 \\
(22.3,24.0)\end{array}$ & $\begin{array}{c}30.2 \\
(29.2,31.1)\end{array}$ & $\begin{array}{c}22.7 \\
(21.8,23.6)\end{array}$ & $\begin{array}{c}15.2 \\
(14.5,16.0\end{array}$ \\
\hline $2008, n=10557$ & $\begin{array}{c}7.8 \\
(7.2,8.4)\end{array}$ & $\begin{array}{c}21.2 \\
(20.4,22.1)\end{array}$ & $\begin{array}{c}29.7 \\
(28.8,30.7)\end{array}$ & $\begin{array}{c}23.9 \\
(23.0,24.8)\end{array}$ & $\begin{array}{c}17.4 \\
(16.6,18.2\end{array}$ \\
\hline \multicolumn{6}{|l|}{ Respondent Age } \\
\hline \multicolumn{6}{|l|}{$65-69$ years* } \\
\hline $1998, n=3018$ & $\begin{array}{c}17.0 \\
(15.5,18.5)\end{array}$ & $\begin{array}{c}30.8 \\
(29.1,32.6)\end{array}$ & $\begin{array}{c}27.0 \\
(25.3,28.8)\end{array}$ & $\begin{array}{c}16.1 \\
(14.7,17.5)\end{array}$ & $\begin{array}{c}9.1 \\
(8.1,10.3)\end{array}$ \\
\hline $2004, n=3392$ & $\begin{array}{c}12.2 \\
(11.0,13.5)\end{array}$ & $\begin{array}{c}26.9 \\
(25.3,28.6)\end{array}$ & $\begin{array}{c}29.4 \\
(27.8,31.1)\end{array}$ & $\begin{array}{c}19.8 \\
(18.3,21.3)\end{array}$ & $\begin{array}{c}11.7 \\
(10.6,12.9\end{array}$ \\
\hline $2008, n=3128$ & $\begin{array}{c}11.7 \\
(10.3,13.2)\end{array}$ & $\begin{array}{c}25.0 \\
(23.2,26.9)\end{array}$ & $\begin{array}{c}30.3 \\
(28.4,32.3)\end{array}$ & $\begin{array}{c}20.4 \\
(18.8,22.1)\end{array}$ & $\begin{array}{c}12.7 \\
(11.4,14.2\end{array}$ \\
\hline \multicolumn{6}{|l|}{ 70-74 years* } \\
\hline $1998, n=2720$ & $\begin{array}{c}14.8 \\
(13.4,16.3)\end{array}$ & $\begin{array}{c}27.7 \\
(26.0,29.5)\end{array}$ & $\begin{array}{c}30.1 \\
(28.3,31.9)\end{array}$ & $\begin{array}{c}17.6 \\
(16.2,19.2)\end{array}$ & $\begin{array}{c}9.8 \\
(8.7,11.0)\end{array}$ \\
\hline $2004, n=2656$ & $\begin{array}{c}10.4 \\
(9.2,11.8)\end{array}$ & $\begin{array}{c}24.3 \\
(22.6,26.2)\end{array}$ & $\begin{array}{c}29.5 \\
(27.6,31.4)\end{array}$ & $\begin{array}{c}21.5 \\
(19.9,23.3)\end{array}$ & $\begin{array}{c}14.2 \\
(12.8,15.8\end{array}$ \\
\hline $2008, n=2908$ & $\begin{array}{c}7.5 \\
(6.5,8.6) \\
\end{array}$ & $\begin{array}{c}21.7 \\
(20.1,23.5) \\
\end{array}$ & $\begin{array}{c}30.8 \\
(28.9,32.7) \\
\end{array}$ & $\begin{array}{c}23.2 \\
(21.5,25.0) \\
\end{array}$ & $\begin{array}{c}16.8 \\
(15.4,18.4 \\
\end{array}$ \\
\hline \multicolumn{6}{|l|}{ 75-79 years* } \\
\hline $1998, n=2284$ & $\begin{array}{c}11.2 \\
(9.9,12.7)\end{array}$ & $\begin{array}{c}24.8 \\
(22.9,26.7)\end{array}$ & $\begin{array}{c}30.0 \\
(28.0,32.1)\end{array}$ & $\begin{array}{c}20.3 \\
(18.6,22.2)\end{array}$ & $\begin{array}{c}13.7 \\
(12.2,15.3\end{array}$ \\
\hline $2004, n=2031$ & $\begin{array}{c}6.4 \\
(5.3,7.6)\end{array}$ & $\begin{array}{c}20.9 \\
(19.0,22.9)\end{array}$ & $\begin{array}{c}32.0 \\
(29.8,34.2)\end{array}$ & $\begin{array}{c}24.1 \\
(22.2,26.2)\end{array}$ & $\begin{array}{c}16.6 \\
(14.9,18.5\end{array}$ \\
\hline $2008, n=2180$ & $\begin{array}{c}5.7 \\
(4.7,6.9) \\
\end{array}$ & $\begin{array}{c}19.3 \\
(17.5,21.3) \\
\end{array}$ & $\begin{array}{c}28.5 \\
(26.4,30.6) \\
\end{array}$ & $\begin{array}{c}26.6 \\
(24.6,28.7) \\
\end{array}$ & $\begin{array}{c}19.9 \\
(18.1,21.9 \\
\end{array}$ \\
\hline \multicolumn{6}{|l|}{$80-84$ years* } \\
\hline $1998, n=1465$ & $\begin{array}{c}8.3 \\
(6.9,10.1)\end{array}$ & $\begin{array}{c}23.7 \\
(21.4,26.2)\end{array}$ & $\begin{array}{c}30.1 \\
(27.6,32.8)\end{array}$ & $\begin{array}{c}22.6 \\
(20.3,25.0)\end{array}$ & $\begin{array}{c}15.3 \\
(13.3,17.5\end{array}$ \\
\hline $2004, n=1609$ & $\begin{array}{c}5.9 \\
(4.8,7.3)\end{array}$ & $\begin{array}{c}19.1 \\
(17.0,21.4)\end{array}$ & $\begin{array}{c}30.3 \\
(27.9,32.9)\end{array}$ & $\begin{array}{c}25.2 \\
(22.9,27.7)\end{array}$ & $\begin{array}{c}19.5 \\
(17.4,21.7\end{array}$ \\
\hline $2008, n=1517$ & $\begin{array}{c}4.6 \\
(3.6,5.9)\end{array}$ & $\begin{array}{c}16.8 \\
(14.9,19.0) \\
\end{array}$ & $\begin{array}{c}30.6 \\
(28.1,33.2)\end{array}$ & $\begin{array}{c}28.1 \\
(25.7,30.7)\end{array}$ & $\begin{array}{c}19.8 \\
(17.7,22.1\end{array}$ \\
\hline \multicolumn{6}{|l|}{$\geq 85$ years* } \\
\hline $1998, n=1306$ & $\begin{array}{c}7.7 \\
(6.1,9.5)\end{array}$ & $\begin{array}{c}22.2 \\
(19.6,24.9)\end{array}$ & $\begin{array}{c}32.0 \\
(29.1,35.0)\end{array}$ & $\begin{array}{c}24.4 \\
(21.8,27.3)\end{array}$ & $\begin{array}{c}13.8 \\
(11.8,16.1)\end{array}$ \\
\hline $\begin{array}{l}2004, n=1425 \\
2008, n=1588\end{array}$ & $\begin{array}{c}5.0 \\
(3.8,6.6) \\
4.5 \\
(4.6,6.2) \\
\end{array}$ & $\begin{array}{c}20.7 \\
(18.3,23.3) \\
17.7 \\
(18.2,21.0) \\
\end{array}$ & $\begin{array}{c}30.0 \\
(27.3,32.9) \\
27.4 \\
(27.8,31.1) \\
\end{array}$ & $\begin{array}{c}26.4 \\
(23.7,29.2) \\
26.3 \\
(24.1,27.2) \\
\end{array}$ & $\begin{array}{c}17.9 \\
(15.6,20.5) \\
24.1 \\
(18.7,21.6\end{array}$ \\
\hline \multicolumn{6}{|l|}{ Respondent Sex } \\
\hline \multicolumn{6}{|l|}{ Male* } \\
\hline $1998, n=4463$ & $\begin{array}{c}13.6 \\
(12.6,14.8)\end{array}$ & $\begin{array}{c}27.2 \\
(25.8,28.6)\end{array}$ & $\begin{array}{c}28.7 \\
(27.3,30.1)\end{array}$ & $\begin{array}{c}19.2 \\
(17.9,20.4)\end{array}$ & $\begin{array}{c}11.3 \\
(10.4,12.4\end{array}$ \\
\hline $2004, n=4595$ & $\begin{array}{c}9.8 \\
(8.9,10.7)\end{array}$ & $\begin{array}{c}22.7 \\
(21.4,24.1)\end{array}$ & $\begin{array}{c}29.2 \\
(27.8,30.7)\end{array}$ & $\begin{array}{c}23.0 \\
(21.7,24.4)\end{array}$ & $\begin{array}{c}15.4 \\
(14.3,16.5\end{array}$ \\
\hline $2008, n=4441$ & $\begin{array}{c}8.9 \\
(7.9,9.9)\end{array}$ & $\begin{array}{c}21.1 \\
(19.8,22.6)\end{array}$ & $\begin{array}{c}28.4 \\
(26.9,30.0)\end{array}$ & $\begin{array}{c}24.1 \\
(22.7,25.5)\end{array}$ & $\begin{array}{c}17.5 \\
(16.3,18.7\end{array}$ \\
\hline
\end{tabular}


Table 2 Percentage of respondents with $0,1,2,3$, or 4 or more chronic diseases by survey year, overall and stratified by age and by sex (Continued)

\begin{tabular}{lccccc}
\hline Female* & \multicolumn{1}{c}{} \\
\hline $1998, n=5927$ & 12.8 & 26.7 & 29.9 & 19.0 & 11.7 \\
$2004, n=6026$ & $(11.9,13.7)$ & $(25.5,27.9)$ & $(28.7,31.2)$ & $(17.9,20.1)$ & $(10.9,12.6)$ \\
& 8.1 & 23.5 & 30.9 & 22.4 & $(15.1$ \\
$2008, n=6116$ & $(7.3,8.8)$ & $(22.3,24.7)$ & $(29.7,32.2)$ & $(21.3,23.6)$ & $(14.2,16.1)$ \\
& $(6.2,7.7)$ & $(20.2,22.5)$ & $(29.5,32.0)$ & $(22.6,25.0)$ & $(16.2,18.4)$ \\
\hline
\end{tabular}

Values are weighted percentages, with $95 \%$ confidence intervals in parentheses, derived by using the HRS respondent population weights of each year to adjust for the sampling design of survey.

* $P$ value $<0.001$, derived from the Wald chi-square tests which were performed to examine group differences in proportions of characteristics across years of survey. P-values refer to whether the proportions or characteristics were significantly different among groups between years.

\section{Sex-Stratified Trends}

For chronic disease trends, the increase in multiple chronic disease prevalence was similar among men and women (Table 2). For impairments, there was a trend towards increasing multiple impairments among women, but not men (Table 3). For disability trends, among both men and women, there were fluctuations in the prevalence of disability but no consistent trends were observed (Table 4).

\section{Sensitivity Analysis}

Removing each individual chronic disease in the count for multiple chronic diseases demonstrated that no individual chronic disease was responsible for the observed trends. For impairment trends, the significant increases in multiple impairments appear to be largely due to the rise in the prevalence in urinary incontinence. Other individual impairments did not account for any significant trends in multiple impairments.

\section{Discussion}

Our analysis of recent concurrent trends in chronic disease, impairment and disability demonstrate that the prevalence of chronic diseases and multiple chronic diseases has increased over time among older adults. In contrast, the prevalence of impairments and multiple impairments, except urinary incontinence, have largely remained stable, as have the prevalence of ADL and IADL disabilities. Overall, despite the increase in the prevalence of chronic disease and multiple chronic diseases, we did not observe a concurrent increase in the prevalence of impairment or disability. Our findings are consistent with older trends, demonstrating the rising prevalence of chronic disease among older adults, as well the relative stability of impairment trends and the declining trends in the prevalence of disability, but clarify that these trends remain consistent when examined concurrently and have persisted as of 2008 .

We also found that there were age and gender disparities in disability trends. Disability for IADL and ADL has declined mostly among the oldest old, and the prevalence of disability was higher in women than men at every time point. For impairments, we observed increases in impairments among the 65-69 age group and among women, which we found were due to the increase in the prevalence of urinary incontinence. Although the overall prevalence of multiple impairments did not increase over time, we observed that the prevalence of multiple impairments remained high particularly among the oldest old group.

Our finding that the prevalence of impairments and disability has largely not been increasing despite growing burden of chronic disease is consistent with prior work on trends. Prior reports have found an increase in chronic disease prevalence throughout the 1990s and 2000s $[4,9]$. Our findings add to this literature not only by examining more recent data, but also by using a single population to also estimate impairment and disability prevalence. That impairment trends were largely stable while chronic disease prevalence increased suggests that chronic diseases were becoming less likely to be associated with impairments over time. Finally, our finding that disability trends were similarly stable is consistent with prior reports which showed that disability has slightly declined over the years, but has been stable more recently $[10,11,14]$.

There are several possible explanations for our finding that impairment and disability patterns over time have not changed significantly despite the dramatic increase in chronic disease prevalence among older adults, particularly given the relationship between chronic diseases and impairments. First, increased screening of chronic disease is likely to identify more disease, but of a less severe variety, such that adults with chronic disease now appear to live with the disease for longer periods of time before causing impairment. Second, either separate from or perhaps partly due to chronic diseases being diagnosed at an earlier stage, chronic diseases may be better managed, reducing the risk of developing impairment from these chronic diseases. For instance, the 


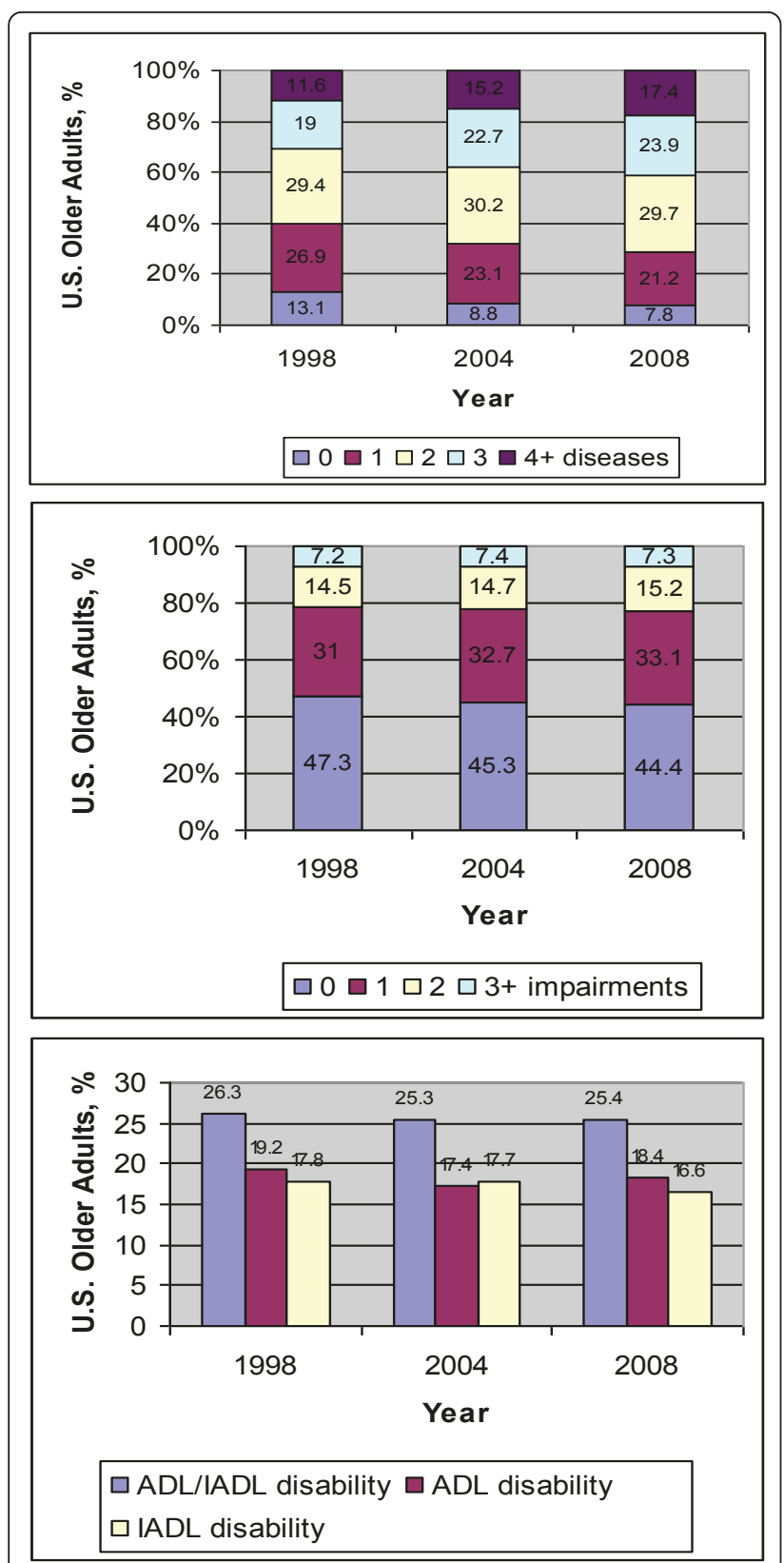

Figure 1 Percentage of older adults aged 65 and over who have $0,1,2,3$, or 4 or more chronic diseases (A), $0,1,2$, or 3 or more impairments (B) and disability in activities in daily living, instrumental activities of daily living or either (C) in the Health and Retirement Study in 1998, 2004 and 2008. All estimates used sampling weights to account for survey design.

availability of greater numbers of pharmaceutical treatments with differing mechanisms of action for coronary heart disease, hypertension, diabetes, COPD and many other diseases over the past 20 years may have improved the management of these diseases among older adults. Examples of diseases which might respond to treatment include arthritis treatment improving mobility; improved treatment of diabetes (better control) may lead to improvement in eye sight, and may also be related to cognition and incontinence. Improved management of chronic lung disease may also improve mobility. Third, improved management of chronic disease and screening for potential sequelae of chronic disease may have reduced the likelihood or impact of impairment arising from disease. An example of this would be improved podiatric care and retinopathy screening among adults with diabetes. Higher education levels over the recent past may also have contributed to greater patient selfseeking of preventive care and improved self-management of chronic disease.

The rise in prevalence in multiple chronic disease among older adults in the U.S. is likely to be associated with rising health care utilization and costs, although thus far, has not been associated with increased rates of impairments and disability. This may seem to suggest that our health care system is working effectively. However, when we consider how the U.S. population is rapidly aging [37], these rates may be misleading. The absolute number of older adults with multiple chronic diseases, impairment and disability all continue to climb even though prevalence rates may be stable for impairments and disability. These trends have substantial ramifications on overall costs incurred by the older adult population. Therefore, it is important to continue to find ways, such as new models of care or reimbursement structures, to effectively manage this expanding population with multiple chronic diseases with substantial burden of disability.

Our study has several strengths. First, we used a large nationally representative database which contains data on chronic diseases, impairments, and disability, allowing for the most up-to-date concurrent examination of national trends to enhance our understanding of health problems faced by older adults today. Due to the aging of the sample in the cohort and replenishment of new birth cohorts, we were able to compare rates of chronic disease, impairments, and disability when the sample was stratified by age and sex. However, our study has limitations as well. First, in order to utilize sampling weights to estimate nationally-representative prevalence rates for all 3 survey waves, we limited our study to community-dwelling respondents and excluded respondents residing in nursing homes. Because the overall size of the nursing home population in the United States has declined over the study period [38], we would expect that adults previously cared for in nursing homes would be more likely to be in the community and thus that prevalence rates of impairment or disability would have risen, rather than remained flat as we observed. Second, our measures of chronic disease, impairment, and disability were based on self-report of conditions. 


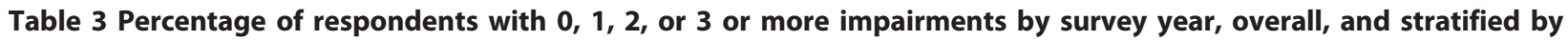
age and by sex

\begin{tabular}{|c|c|c|c|c|}
\hline & \multicolumn{4}{|c|}{ Number of Impairments, \% (95\% Confidence Interval) } \\
\hline & 0 & 1 & 2 & $3+$ \\
\hline \multicolumn{5}{|l|}{ Overall* } \\
\hline $1998, n=10390$ & $\begin{array}{c}47.3 \\
(46.3,48.4)\end{array}$ & $\begin{array}{c}31.0 \\
(30.0,31.9)\end{array}$ & $\begin{array}{c}14.5 \\
(13.8,15.3)\end{array}$ & $\begin{array}{c}7.2 \\
(6.7,7.7)\end{array}$ \\
\hline $2004, n=10621$ & $\begin{array}{c}45.3 \\
(44.2,46.3)\end{array}$ & $\begin{array}{c}32.7 \\
(31.6,33.7)\end{array}$ & $\begin{array}{c}14.7 \\
(13.9,15.4)\end{array}$ & $\begin{array}{c}7.4 \\
(6.9,8.0)\end{array}$ \\
\hline $2008, n=10557$ & $\begin{array}{c}44.4 \\
(43.3,45.5)\end{array}$ & $\begin{array}{c}33.1 \\
(32.1,34.1)\end{array}$ & $\begin{array}{c}15.2 \\
(14.5,16.0)\end{array}$ & $\begin{array}{c}7.3 \\
(6.8,7.9)\end{array}$ \\
\hline \multicolumn{5}{|l|}{ Respondent Age } \\
\hline \multicolumn{5}{|l|}{$65-69$ yearst } \\
\hline $1998, n=3018$ & $\begin{array}{c}58.8 \\
(56.9,60.7)\end{array}$ & $\begin{array}{c}28.6 \\
(26.9,30.4)\end{array}$ & $\begin{array}{c}9.1 \\
(8.0,10.2)\end{array}$ & $\begin{array}{c}3.6 \\
(2.9,4.3)\end{array}$ \\
\hline $2004, n=3392$ & $\begin{array}{c}55.7 \\
(53.8,57.5)\end{array}$ & $\begin{array}{c}30.1 \\
(28.4,31.8)\end{array}$ & $\begin{array}{c}10.2 \\
(9.1,11.4)\end{array}$ & $\begin{array}{c}4.1 \\
(3.4,4.9)\end{array}$ \\
\hline $2008, n=3128$ & $\begin{array}{c}53.0 \\
(50.9,55.1) \\
\end{array}$ & $\begin{array}{c}32.2 \\
(30.3,34.2) \\
\end{array}$ & $\begin{array}{c}11.2 \\
(9.9,12.6) \\
\end{array}$ & $\begin{array}{c}3.6 \\
(2.9,4.5) \\
\end{array}$ \\
\hline \multicolumn{5}{|l|}{ 70-74 years } \\
\hline $1998, n=2720$ & $\begin{array}{c}51.4 \\
(49.3,53.3)\end{array}$ & $\begin{array}{c}31.5 \\
(29.6,33.3)\end{array}$ & $\begin{array}{c}12.4 \\
(11.1,13.7)\end{array}$ & $\begin{array}{c}4.7 \\
(4.1,5.8)\end{array}$ \\
\hline $2004, n=2656$ & $\begin{array}{c}49.2 \\
(47.1,51.2)\end{array}$ & $\begin{array}{c}32.9 \\
(30.9,34.9)\end{array}$ & $\begin{array}{c}12.5 \\
(11.2,14.0)\end{array}$ & $\begin{array}{c}5.4 \\
(4.6,6.5)\end{array}$ \\
\hline $2008, n=2908$ & $\begin{array}{c}49.1 \\
(47.1,51.2) \\
\end{array}$ & $\begin{array}{c}32.7 \\
(30.9,34.7) \\
\end{array}$ & $\begin{array}{c}13.5 \\
(12.1,14.9) \\
\end{array}$ & $\begin{array}{c}4.7 \\
(3.9,5.6) \\
\end{array}$ \\
\hline \multicolumn{5}{|l|}{$75-79$ years } \\
\hline $1998, n=2284$ & $\begin{array}{c}44.5 \\
(42.2,46.7)\end{array}$ & $\begin{array}{c}33.4 \\
(31.3,35.5)\end{array}$ & $\begin{array}{c}15.2 \\
(13.6,16.8)\end{array}$ & $\begin{array}{c}7.1 \\
(6.0,8.3)\end{array}$ \\
\hline $2004, n=2031$ & $\begin{array}{c}43.2 \\
(40.9,45.6)\end{array}$ & $\begin{array}{c}35 \\
(32.7,37.2)\end{array}$ & $\begin{array}{c}15.6 \\
(14.0,17.4)\end{array}$ & $\begin{array}{c}6.3 \\
(5.3,7.5)\end{array}$ \\
\hline $2008, n=2180$ & $\begin{array}{c}41.2 \\
(38.9,43.5)\end{array}$ & $\begin{array}{c}36.5 \\
(34.3,38.8) \\
\end{array}$ & $\begin{array}{c}15.3 \\
(13.7,17.1) \\
\end{array}$ & $\begin{array}{c}7 \\
(5.8,8.3) \\
\end{array}$ \\
\hline \multicolumn{5}{|l|}{$80-84$ years } \\
\hline $1998, n=1465$ & $\begin{array}{c}35.1 \\
(32.4,37.9)\end{array}$ & $\begin{array}{c}32.8 \\
(30.2,35.6)\end{array}$ & $\begin{array}{c}21.5 \\
(19.2,23.9)\end{array}$ & $\begin{array}{c}10.6 \\
(9.0,12.5)\end{array}$ \\
\hline $2004, n=1609$ & $\begin{array}{c}38.1 \\
(35.5,40.8)\end{array}$ & $\begin{array}{c}35.2 \\
(32.7,37.9)\end{array}$ & $\begin{array}{c}17.1 \\
(15.1,19.2)\end{array}$ & $\begin{array}{c}9.6 \\
(8.2,11.3)\end{array}$ \\
\hline $2008, n=1517$ & $\begin{array}{c}36.3 \\
(33.7,39.1)\end{array}$ & $\begin{array}{c}34.7 \\
(32.1,37.4)\end{array}$ & $\begin{array}{c}18.7 \\
(16.7,20.9)\end{array}$ & $\begin{array}{c}10.2 \\
(8.7,12.0)\end{array}$ \\
\hline \multicolumn{5}{|l|}{$\geq 85$ years } \\
\hline $1998, n=1306$ & $\begin{array}{c}24.1 \\
(21.5,27.0)\end{array}$ & $\begin{array}{c}28.4 \\
(25.6,31.3)\end{array}$ & $\begin{array}{c}26.4 \\
(23.8,29.3)\end{array}$ & $\begin{array}{c}21 \\
(18.6,23.8)\end{array}$ \\
\hline $2004, n=1425$ & $\begin{array}{c}23.6 \\
(21.0,26.3)\end{array}$ & $\begin{array}{c}30.9 \\
(28.1,33.8)\end{array}$ & $\begin{array}{c}25.8 \\
(23.2,28.6)\end{array}$ & $\begin{array}{c}19.8 \\
(17.4,22.4\end{array}$ \\
\hline $2008, n=1588$ & $\begin{array}{c}25.5 \\
(23.0,28.2)\end{array}$ & $\begin{array}{c}29.5 \\
(26.9,32.2)\end{array}$ & $\begin{array}{c}25.5 \\
(23.0,28.2)\end{array}$ & $\begin{array}{c}19.5 \\
(17.3,21.9\end{array}$ \\
\hline \multicolumn{5}{|l|}{ Respondent Sex } \\
\hline \multicolumn{5}{|l|}{ Male } \\
\hline $1998, n=4463$ & $\begin{array}{c}47.5 \\
(45.9,49.1)\end{array}$ & $\begin{array}{c}32.2 \\
(30.7,33.7)\end{array}$ & $\begin{array}{c}14.2 \\
(13.1,15.3)\end{array}$ & $\begin{array}{c}6.2 \\
(5.5,6.9)\end{array}$ \\
\hline $2004, n=4595$ & $\begin{array}{c}47.5 \\
(45.9,49.0)\end{array}$ & $\begin{array}{c}31.9 \\
(30.4,33.4)\end{array}$ & $\begin{array}{c}14.4 \\
(13.3,15.5)\end{array}$ & $\begin{array}{c}6.3 \\
(5.6,7.1)\end{array}$ \\
\hline $2008, n=4441$ & $\begin{array}{c}47.8 \\
(46.1,49.5)\end{array}$ & $\begin{array}{c}32.3 \\
(30.7,33.8)\end{array}$ & $\begin{array}{c}14.5 \\
(13.3,15.7)\end{array}$ & $\begin{array}{c}5.5 \\
(4.8,6.2)\end{array}$ \\
\hline
\end{tabular}




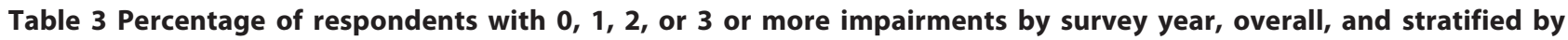
age and by sex (Continued)

\begin{tabular}{|c|c|c|c|c|}
\hline Female* & & & & \\
\hline $1998, n=5927$ & $\begin{array}{c}47.2 \\
(46.9,48.6)\end{array}$ & $\begin{array}{c}30.1 \\
(28.9,31.4)\end{array}$ & $\begin{array}{c}14.8 \\
(13.8,15.8)\end{array}$ & $\begin{array}{c}7.9 \\
(7.2,8.7)\end{array}$ \\
\hline $2004, n=6026$ & $\begin{array}{c}43.6 \\
(42.3,45.0)\end{array}$ & $\begin{array}{c}33.3 \\
(32.0,34.6)\end{array}$ & $\begin{array}{c}14.9 \\
(13.9,15.9)\end{array}$ & $\begin{array}{c}8.3 \\
(7.6,9.1)\end{array}$ \\
\hline $2008, n=6116$ & $\begin{array}{c}41.8 \\
(40.4,43.2)\end{array}$ & $\begin{array}{c}33.7 \\
(32.4,35.0)\end{array}$ & $\begin{array}{c}15.8 \\
(14.8,16.9)\end{array}$ & $\begin{array}{c}8.7 \\
(7.9,9.5)\end{array}$ \\
\hline
\end{tabular}

Values are weighted percentages, with $95 \%$ confidence intervals in parentheses, derived by using the HRS respondent population weights of each year to adjust for the sampling design of survey.

* $\mathrm{P}$ value $<0.001$, derived from the Wald chi-square tests which were performed to examine group differences in proportions of characteristics across years of survey. P-values refer to whether the proportions or characteristics were significantly different among groups between years.

$\dagger \mathrm{P}$ value $=0.01$.

Table 4 Percentage of respondents with disability in activities of daily living (ADL), instrumental activities of daily living (IADL) and either ADL or IADL, stratified by age and by sex

\begin{tabular}{|c|c|c|c|}
\hline & \multicolumn{3}{|c|}{ ADL or IADL Disability, \% (95\% Confidence Interval) } \\
\hline & Any ADL & Any IADL & Either ADL or IADL \\
\hline \multicolumn{4}{|l|}{ Overall } \\
\hline $1998, n=10390$ & $19.2(18.4,20.0)$ & $17.8(17.0,18.6)$ & $26.3(25.4,27.2)$ \\
\hline $2004, n=10621$ & $17.4(16.6,18.2)$ & $17.7(16.9,18.5)$ & $25.3(24.4,26.2)$ \\
\hline \multirow[t]{2}{*}{$2008, n=10557$} & $18.4(17.6,19.2)$ & $16.6(15.8,17.4)$ & $25.4(24.5,26.3)$ \\
\hline & $p=0.01$ & $p=0.06$ & $p=0.25$ \\
\hline \multicolumn{4}{|l|}{ Respondent Age } \\
\hline \multicolumn{4}{|l|}{$65-69$ years } \\
\hline $1998, n=3018$ & $12.4(11.2,13.7)$ & $9.1(8.0,10.2)$ & $16.3(14.9,17.8)$ \\
\hline $2004, n=3392$ & $11.3(10.2,12.6)$ & $9.2(8.2,10.4)$ & $15.5(14.2,16.9)$ \\
\hline \multirow[t]{2}{*}{$2008, n=3128$} & $13.1(11.7,14.7)$ & $9.5(8.3,10.8)$ & $17.0(15.5,18.7)$ \\
\hline & $p=0.15$ & $p=0.89$ & $p=0.31$ \\
\hline \multicolumn{4}{|l|}{ 70-74 years } \\
\hline $1998, n=2720$ & $15.3(13.9,16.8)$ & $12.6(11.0,13.5)$ & $20.4(18.8,22.0)$ \\
\hline \multirow[t]{2}{*}{$\begin{array}{l}2004, n=2656 \\
2008, n=2908\end{array}$} & $\begin{array}{l}13.4(12.0,14.9) \\
13.2(11.9,14.6)\end{array}$ & $\begin{array}{l}12.2(10.9,13.6) \\
11.7(10.5,13.1)\end{array}$ & $\begin{array}{l}19(17.4,20.7) \\
19(17.4,20.6)\end{array}$ \\
\hline & $p=0.08$ & $p=0.84$ & $P=0.38$ \\
\hline \multicolumn{4}{|l|}{ 75-79 years } \\
\hline $1998, n=2284$ & $19.4(17.7,21.3)$ & $19.1(17.4,20.9)$ & $27.6(25.6,29.6)$ \\
\hline $2004, n=2031$ & $17.1(15.3,18.9)$ & $17.6(15.8,19.4)$ & $25.7(23.7,27.8)$ \\
\hline \multirow[t]{2}{*}{$2008, n=2180$} & $19.2(17.4,21.2)$ & $15.1(13.4,16.8)$ & $25.4(23.4,27.5)$ \\
\hline & $p=0.12$ & $p=0.01$ & $p=0.27$ \\
\hline \multicolumn{4}{|l|}{$80-84$ years } \\
\hline $1998, n=1465$ & $26.5(24.1,29.1)$ & $26.7(24.2,29.2)$ & $37.6(34.9,40.4)$ \\
\hline $2004, n=1609$ & $22.2(20.1,24.5)$ & $23.4(21.1,25.8)$ & $32.8(30.3,35.4)$ \\
\hline \multirow[t]{2}{*}{$2008, n=1517$} & $23.1(22.4,25.2)$ & $24.6(22.3,27.1)$ & $35.1(32.5,37.8)$ \\
\hline & $p=0.03$ & $p=0.17$ & $p=0.05$ \\
\hline \multicolumn{4}{|l|}{$\geq 85$ years } \\
\hline $1998, n=1306$ & $41(38.0,44.2)$ & $46.6(43.3,49.6)$ & $56.4(53.2,59.5)$ \\
\hline $2004, n=1425$ & $36.6(33.7,39.3)$ & $44.7(41.5,47.7)$ & $54.0(50.9,57.1)$ \\
\hline \multirow[t]{2}{*}{$2008, n=1588$} & $36.4(35.9,39.4)$ & $39.1(41.0,44.5)$ & $50.2(47.2,53.1)$ \\
\hline & $p=0.08$ & $p=0.002$ & $p=0.02$ \\
\hline
\end{tabular}


Table 4 Percentage of respondents with disability in activities of daily living (ADL), instrumental activities of daily living (IADL) and either ADL or IADL, stratified by age and by sex (Continued)

\begin{tabular}{|c|c|c|c|}
\hline Respondent Sex & & & \\
\hline \multicolumn{4}{|l|}{ Male } \\
\hline $1998, n=4463$ & $15.6(14.5,16.8)$ & $15.3(14.2,16.5)$ & $23.0(21.7,24.3)$ \\
\hline $2004, n=4595$ & $14.9(13.9,16.1)$ & $15.9(14.7,17.0)$ & $23.2(21.9,24.5)$ \\
\hline \multirow[t]{2}{*}{$2008, n=4441$} & $15.0(13.9,16.2)$ & $13.8(12.7,14.9)$ & $21.6(20.3,23.0)$ \\
\hline & $p=0.65$ & $p=0.02$ & $p=0.18$ \\
\hline \multicolumn{4}{|l|}{ Female } \\
\hline $1998, n=5927$ & $21.7(20.6,22.9)$ & $19.6(18.6,20.7)$ & $28.7(27.5,30.0)$ \\
\hline $2004, n=6026$ & $19.3(18.3,20.4)$ & $19.1(18.0,20.2)$ & $26.9(25.7,28.2)$ \\
\hline \multirow[t]{2}{*}{$2008, n=6116$} & $21.0(19.8,22.1)$ & $18.8(17.7,19.9)$ & $28.3(27.0,29.5)$ \\
\hline & $p=0.01$ & $p=0.53$ & $p=0.11$ \\
\hline
\end{tabular}

Values are weighted percentages, with $95 \%$ confidence intervals in parentheses, derived by using the HRS respondent population weights of each year to adjust for the sampling design of survey.

Note: $\mathrm{P}$ values were derived from the Wald chi-square tests which were performed to examine group differences in proportions of characteristics across years of survey. P-values refer to whether the proportions of characteristics were significantly different among groups between years.

The HRS provides unique longitudinal survey data to identify population health trends. In population-based cohorts, self-reporting of health conditions is an accepted methodology for large, nationally representative survey for which detailed chart review is not feasible and the concordance between self report and medical record review is generally good $(\kappa=0.60)$ [39]. Although the survey is limited by its use of self report to ascertain chronic disease, impairment and disability, prior studies have suggested that self-report provides accurate prevalence estimates for all three [39-44].

\section{Conclusions}

A main goal of successful aging is the maintenance of independence [45]. In fact, a main aspect of the health care of older adults is the attention paid to maintain functional ability; preventing disability by the effective management of chronic diseases and impairments is of paramount importance to the care of older adults so that they can continue to live independently in the community for as long as possible. Our study shows that multiple chronic diseases have become increasingly prevalent and impairments and disability continue to be a substantial, although not rising, burden among older adults. The aging, U.S. population continues to need high-quality care, focused on managing multiple chronic diseases and preventing impairment and disability.

\section{Abbreviations \\ (ADL): Activities of daily living; (BMI): body mass index; (Cl): confidence interval; (COPD): chronic obstructive pulmonary disease; (HRS): Health and Retirement Study; (IADL): instrumental activities of daily living.}

\section{Acknowledgements}

$\mathrm{WH}$ is a John A Hartford Foundation Center of Excellence Scholar and a New York Academy of Medicine Hoar Fellow. JR is currently supported by the National Institute on Aging (K08 AG032886) and by the American
Federation of Aging Research through the Paul B. Beeson Career Development Award Program. The Health and Retirement Study is sponsored by the National Institute on Aging (grant number U01AG009740) and conducted by the Institute for Social Research at the University of Michigan.

\section{Author details}

${ }^{1}$ Department of Geriatrics and Palliative Medicine, Mount Sinai School of Medicine, New York, NY, USA. ${ }^{2}$ The Health Services Research and

Development Research Enhancement Award Program and the Geriatrics Research, Education, and Clinical Center, James J. Peters Veterans Affairs Medical Center, Bronx, NY, USA. ${ }^{3}$ Division of General Internal Medicine, Yale University School of Medicine and Center for Outcomes Research and Evaluation, Yale-New Haven Hospital, New Haven, CT, USA.

\section{Authors' contributions}

Specific contributions by individual authors are described below: Study Concept and design: WH, JR, AS; Acquisition of Data: WH; Analysis and interpretation of data: $\mathrm{WH}, J \mathrm{R}, \mathrm{KB}, \mathrm{AS}$; Drafting of the manuscript: $\mathrm{WH}, \mathrm{JR}$; Critical revision of the manuscript for important intellectual content: $\mathrm{WH}, J \mathrm{R}, \mathrm{KB}$, AS; Statistical Analysis: WH; Study Supervision: JR, AS. All authors read and approved the final manuscript.

\section{Competing interests}

The authors declare that they have no competing interests.

Received: 10 February 2011 Accepted: 18 August 2011 Published: 18 August 2011

\section{References}

1. Verbrugge LM, Jette AM: The disablement process. Soc Sci Med 1994, 38(1):1-14.

2. Nagi SZ: The concept and measurement of disability. In Disability Policies and Government Programs. Edited by: Berkowiez ED. Praeger, New York; 1979:1-15

3. Grimby G, Finnstam J, Jette A: On the Application of the WHO handicap classification in rehabilitation. Scand J Rehab Med 1988, 20:93-98.

4. Thorpe KE, Howard DH: The rise in spending among Medicare Beneficiaries: the role of chronic disease prevalence and changes in treatment intensity. Health Affairs 2006, 25(5):378-88.

5. Freedman VA, Martin LG: Contribution of chronic conditions to aggregate changes in old-age functioning. Am J Public Health 2000, 90:1755-1760

6. Wolff $J$, Starfield B, Anderson G: Prevalence, expenditures and complications of multiple chronic conditions in the elderly. Arch Intern Med 2002, 162:2269-2276.

7. Vogeli C, Shields AE, Lee TA, Gibson TB, Marder WD, Weiss KB, Blumenthal D: Multiple chronic conditions: prevalence, health 
consequences, and implications for quality, care management and costs. JGIM 2007, 22(S3):391-395.

8. Manton KG, Gu XL: Changes in the prevalence of chronic disability in the United States black and nonblack population above age 65 from 1982 to 1999. PNAS 2001, 98:6354-6359.

9. Manton KG, Corder L, Stallard E: Chronic disability trends in elderly United States populations: 1982-1994. Proc Natl Acad Sci 1997, 94:2593-2598.

10. Manton KG, Gu XL, Lamb VL: Change in chronic disability from 1982 to 2004/2005 as measured by long-term changes in function and health in the U.S. elderly population. PNAS 2006, 103:18374-18379.

11. Schoeni R, Freedman V, Martin L: Why is late-life disability declining? The Milbank Quarterly 2008, 86(1):47-89.

12. Seeman TE, Merkin SS, Crimmins EM, Karlamangla AS: Disability trends among older Americans: National Health and Nutrition Examination Surveys, 1988-1994 and 1999-2004. American Journal of Public Health 2010, 100(1):100-107.

13. Freedman VA, Martin LG, Schoeni RF, Cornman JC: Declines in late-life disability: the role of early- and mid-life factors. Soc Sci Med 2008, 66:1588-1602.

14. Schoeni R, Freedman VA, Wallace R: Persistent, consistent widespread and robust? Another look at recent trends in old-age disability. J Gerontol $B$ Psychol Sci Soc Sci 2001, 56:S206-218.

15. Liao Y, McGee DL, Cao G, Cooper RS: Recent changes in the health status of the older US population: findings from the 1984 and 1994 supplement on aging. J Am Geriatr Soc 2001, 49:443-449.

16. Crimmins EM, Saito Y: Change in the prevalence of diseases among older Americans: 1984-1994. Demographic Res 2000, 9:1-20.

17. Liao Y, McGee DL, Cao F, Cooper RS: Quality of the last year of life of older adults: 1986 vs 1993. JAMA 2000, 283:512-518.

18. Puts MT, Deeg DJ, Hoeymans N, Nusselder WJ, Schellevis FG: Changes in the prevalence of chronic disease and the association with disability in the older Dutch population between 1987 and 2001. Age Ageing 2008, 37(2):187-93.

19. van Gool CH, Picavet HS, Deeg DJ, de Klerk MM, Nusselder WJ, van Boxtel MP, Wong A, Hoeymans N: Trends in activity limitations: the Dutch older population between 1990 and 2007. Int J Epidemiol 2011.

20. Organization for Economic Co-operation and Development (OECD): Trends in Severe Disability Among Elderly People: Assessing the Evidence in 12 OECD Countries and the Future Implications. Paris: OECD Health working papers; 2007, 26

21. Desai M, Pratt LA, Lentzner $H$, Robinson $K N$ : Trends in vision and hearing among older Americans. Hyattsville. MD: National center for Health Statistics; 2001, Aging Trends, No. 2

22. Davila EP, Caban-Martinez AJ, Muennig P, Lee DJ, Fleming LE, Ferraro KF, LeBlanc WG, Lam BL, Arheart KL, McCollister KE, Zheng D, Christ SL: Sensory impairment among older US workers. Am J Public Health 2009, 99(8):1378-85.

23. Freedman VA, Aykan $H$, Martin LG: Aggregate changes in severe cognitive impairment among older Americans: 1993 and 1998. J Gerontol B Psychol Sci Soc Sci 2001, 56:S100-111.

24. Freedman VA, Martin LG, Schoeni RF: Recent trends in disability and functioning among older adults in the United States. JAMA 2002, 288:3137-3146.

25. Rodgers WL, Ofstedal MB, Herzog AR: Trends in scores on tests of cognitive ability in the elderly US population, 1993-2000. Journal of Gerontol Soc Sci 2003, 58:S338-46.

26. Juster FT, Suzman R: An overview of the Health and Retirement Study. Hum Resour 1995, 30:S7-S56.

27. Soldo BJ, Hurd MD, Rodgers WL, Wallace RB: Asset and health dynamics among the oldest old: an overview of the AHEAD Study. J Gerontol B Psychol Sci Soc Sci 1997, 52:S1-20.

28. Heeringa S: Technical description of the asset and health dynamics survey sample design. Ann Arbor, Ml: Institute for social Research, University of Michigan; 1995.

29. Cigolle C, Langa KM, Kabeto MU, Tian Z, Blaum CS: Geriatric conditions and disability: the Health and Retirement Study. Annals of Interna Medicine 2007, 147(3):156-164.

30. Herzog A, Wallace RB: Measures of cognitive functioning in the AHEAD study. J Gerontol B Psychol Sci Soc Sci 1997, , 52B: 37-48.
31. Folstein MF, Folstein SE, Mchugh PR: Mini- mental state. A practical method for grading the cognitive state of patients for the clinician. Psychiatr Res 1975, 12:189-98.

32. Langa KM, Larson EB, Karlawish J, Cutler DM, Kabeto MU, Kim SY, Rosen AB: Trends in the prevalence and mortality of cognitive impairment in the United States: Is there evidence of a compression of cognitive morbidity? Alzheimer's \& Dementia 4(2):134-144.

33. Langa KM, Chernew ME, Kabeto MU, Herzog AR, Ofstedal MB, Willis RJ, Wallace RB, Mucha LM, Straus WL, Fendrick AM: National estimates of the quantity and cost of informal caregiving for the elderly with dementia. $J$ Gen Intern Med 2001, 56:M146-156.

34. Anger JT, Saigal CS, Litwin MS: The Prevalence of Urinary Incontinence Among Community Dwelling Adult Women: Results From the National Health and Nutrition Examination Survey. J Urol 2006, 175(2):601-4.

35. Rivera JA, Fried LP, Weiss CO, Simonsick EM: At the tipping point: predicting severe mobility difficulty in vulnerable older women. J Am Geriatr Soc 2008, 56(8):1417-23.

36. Sampling weights revised for Tracker 2.0 and beyond. HRS staff 2004 [http://hrsonline.isr.umich.edu/sitedocs/wghtdoc.pdf].

37. U.S, Census Bureau, National Population Projections: Table 2a. Projected population of the United States, by age and sex: 2000-2050; [http://www. census.gov/population/www/projections/usinterimproj/].

38. National Center for Health Statistics: Health, United States 2009 With Special Feature on Medical Technology. Hyattsville, MD; 2010, 389, Table 119.

39. Tisnado DM, Adams IL, Liu H, Damberg CL, Chen WP, Hu FA, Carlisle DM, Mangione $\mathrm{CM}$, Kahn KL: What is the concordance between the medical record and patient self-report as data sources for ambulatory care? Med Care 2006, 44:132-140.

40. Mangione CM, Lee PP, Pitts J, Guti'errez P, Berry S, Hays RD: Psychometric properties of the National Eye Institute Visual Function Questionnaire (NEI-VFQ). NEI-VFQ field test investigators. Arch Ophthalmol 1998, 116(11):1496-1504.

41. Mangione CM, Lee PP, Guti'errez PR, Spritzer K, Berry S, Hays RD: Development of the 25-item National Eye Institute Visual Function Questionnaire. Arch Ophthalmol 2001, 119(7):1050-1058.

42. Sindhusake D, Mitchell P, Smith W, Golding M, Newall P, Hartley D, Rubin G: Validation of self-reported hearing loss. The Blue Mountains Hearing Study. Int J Epidemiol 2001, 30(6):1371-8.

43. Elam JT, Graney MJ, Beaver T, Detwi DE, Applegate WB, Miller ST: Comparison of subjective ratings of function with observed functional ability of frail older persons. Am J Pub Health 1991, 81(9):1127-1130.

44. Diokno AC, Brown MB, Brock BM, Herzog AR, Normolle DP: Clinical and cystometric characteristics of continent and incontinent noninstitutionalized elderly. J Urol 1988, 140:567.

45. Depp CA, Jeste DV: Definitions and predictors of successful aging: comprehensive review of larger quantitative studies. Am J Geriatr Psychiatry 2006, 14(1):6-20.

\section{Pre-publication history}

The pre-publication history for this paper can be accessed here: http://www.biomedcentral.com/1471-2318/11/47/prepub

doi:10.1186/1471-2318-11-47

Cite this article as: Hung et al:: Recent trends in chronic disease, impairment and disability among older adults in the United States. BMC Geriatrics 2011 11:47. 